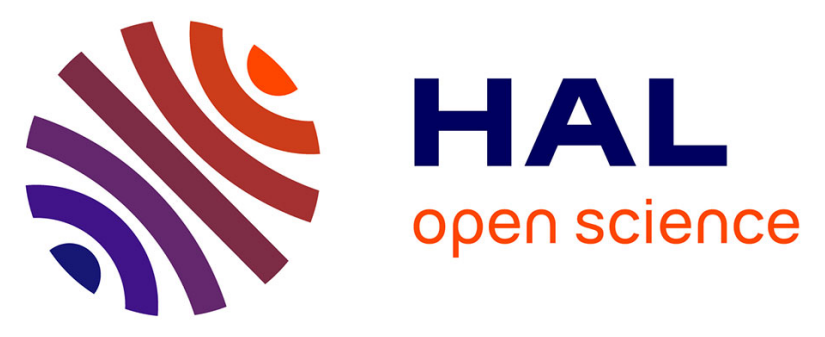

\title{
Influence of Depth Cues on Eye Tracking Depth Measurement in Augmented Reality Using the Magic Leap Device
}

Pierre Raimbaud, Maria Sofia Alvarez Lopez, Pablo Figueroa, Jose Tiberio Hernandez

\section{To cite this version:}

Pierre Raimbaud, Maria Sofia Alvarez Lopez, Pablo Figueroa, Jose Tiberio Hernandez. Influence of Depth Cues on Eye Tracking Depth Measurement in Augmented Reality Using the Magic Leap Device. 2020 IEEE Conference on Virtual Reality and 3D User Interfaces - 6th Annual Workshop on Perceptual and Cognitive Issues in AR, Mar 2020, Atlanta, United States. pp.210-214, 10.1109/VRW50115.2020.00042 . hal-02518855

\section{HAL Id: hal-02518855 \\ https://hal.science/hal-02518855}

Submitted on 25 Mar 2020

HAL is a multi-disciplinary open access archive for the deposit and dissemination of scientific research documents, whether they are published or not. The documents may come from teaching and research institutions in France or abroad, or from public or private research centers.
L'archive ouverte pluridisciplinaire HAL, est destinée au dépôt et à la diffusion de documents scientifiques de niveau recherche, publiés ou non, émanant des établissements d'enseignement et de recherche français ou étrangers, des laboratoires publics ou privés. 


\title{
Influence of Depth Cues on Eye Tracking Depth Measurement in Augmented Reality Using the MagicLeap Device
}

\author{
Pierre Raimbaud* \\ Arts et Metiers Institute of Technology, LISPEN, HESAM Université, F-71100 Chalon-Sur-Saône, France \\ Systems and Computing Engineering, Universidad de los Andes, Bogota D.C., Colombia \\ Maria Sofia Alvarez Lopez ${ }^{\dagger} \quad$ Pablo Figueroa ${ }^{\ddagger}$ Jose Tiberio Hernandez ${ }^{\S}$ \\ Systems and Computing Engineering, Universidad de los Andes, Bogota, D.C., Colombia
}

\begin{abstract}
The eye-tracking technology is currently implemented in many mixed reality devices. However, eye-tracking measurements must be precise enough to permit correct localization in the environment; to allow, for example, linking such spatial positions to virtual objects. According to the human vision, major measurement issues would more likely occur in the depth axis rather than in the horizontal and vertical axes. In the literature, depth cues are known for improving human depth perception. In this study, our hypothesis is that, in an augmented reality environment, the more realistic virtual objects are displayed, thanks to depth cues, the more precise the eye-tracking device depth measures would be. Thus, using the MagicLeap device, we studied the effects of lighting and textures on eye-tracking depth measurement precision, by comparing the measures obtained under varying conditions of lights and textures, on both real and virtual objects. The results confirmed our general hypothesis, and we noticed a more significant influence of lights rather than textures on the precision of the measures. Moreover, we found that these depth cues reduce the measurement imprecision among observers, making the eye-tracking system more accurate when measuring depth.
\end{abstract}

Index Terms: Human-centered computing-Visualization techniques-Computer graphics-Graphics systems and interfaces;

\section{INTRODUCTION}

Depth perception has been studied in different fields, from the computer science itself [21] [22] [8] to the medical sciences [5]. As well as in virtual reality, [13] distance perception has been addressed as an important issue in augmented reality [24]. Because of this, in the (more generic) mixed reality field, previous research on depth perception has mainly focused on the human perception of depth [7]. In this sense, such studies aim to either measure the users' subjective depth perception and compare it for several distances, or to evaluate the efficiency of the techniques for compensating the subjective overestimation or underestimation of the human depth perception.

Nonetheless, with the emergence of eye-tracking technologies in mixed reality devices, vision focus can now be measured in terms of 3D spatial positions. Thus, these eye-tracking measures can offer an objective evaluation of depth, in spite of the devicerelated imprecision. In this way, this paper aims to evaluate if, in the same way that human subjective depth perception can be improved thanks to the presence of depth cues, the latter can also increase the precision of depth measures coming from an eye-tracking device, in this experiment, the Magic Leap, an augmented reality head-

\footnotetext{
*e-mail: pierre.raimbaud@ensam.eu

†e-mail: ms.alvarezl@uniandes.edu.co

†e-mail: pfiguero@uniandes.edu.co

§e-mail: jhernand@uniandes.edu.co
}

mounted display. This hypothesis arises from the fact that even if the measures come from a device, the computation done is based on the human physical reaction of each eye to the objects displayed in the environment. To sum up, this study aims to evaluate the influence of depth cues - in this case, lighting and texture- on eye-tracking measurement precision, in an augmented reality environment.

\section{Related WORK}

First, about depth perception, it should be noted that humans usually divide their environment into three subspaces: the personal space (up to $2 \mathrm{~m}$ ), the action space (between 2 and $30 \mathrm{~m}$ ) and the distant space (beyond $30 \mathrm{~m}$ ) [6]. Moreover, research on distance estimation usually classifies distances as egocentric or exocentric. Note that studies in augmented reality typically focus on egocentric distance, i.e. between the observer and one object in his field of regard [17]. This could be a consequence of the small field of view in the current devices. Moreover, in regards of depth perception in mixed reality, observers tend to underestimate objects' distance in the action and distant space, while they overestimate it for objects in the personal space [9]. In the absence of visual aids, humans tend to estimate distance to objects with an egocentric distance of $0.9 \pm 0.2 \mathrm{~m} \mathrm{[12].}$

In the literature, visual aids for depth perception are called depth cues. These play a crucial role in the success or failure of interpreting augmented content [13], and can be classified as visual or oculomotor depth cues [20]. Here our research was based on the studies that focused on the visual rather than the oculomotor depth cues. Nonetheless, some studies showed that oculomotor depth cues, such as vergence and accommodation, can improve depth perception significantly [1]. Visual depth cues, on the other hand, can be classified as binocular and monocular. Literature regarding augmented reality showed that, in the personal space, binocular cues (such as stereopsis and eye convergence) have a more significant effect than the monocular ones [6]. More precisely, Schmidt et al. [21] studied how the spatial relationships between the user and the real world objects can be modified thanks to perceptual illusions (such as color temperature, or binocular disparity) and found that binocular vision dominated all the other cues. Nonetheless, despite of what was previously stated, monocular cues are, still, of special importance. Indeed, Schmidt et al. noticed in another study [22], that brightness differences, a monocular cue, can highly improve depth perception. Some other monocular cues are shadows, texture and focal depth [21] [18].

Because of being so numerous, in a real environment, there is rarely only one depth cue as visual aid. However, in mixed reality, design choices that impact depth cues can be made [7], allowing the study of their effect. Accordingly, Mikkola et al. [19] compared the effect of some binocular and monocular depth cues for an autostereoscopic display, and they showed that binocular cues tend to have a more significant effect on depth perception ; yet, regarding monocular cues, shadows and texture had a more significant effect than focal depth. Similarly, Wanger et al. [25] evaluated casting shadows, object texture, ground texture and elevation effects on the performance of object manipulations tasks, such as positioning [4]. 
Results showed that shadows had a higher effect than the other evaluated cues on enhancing the performance in the depth axis. Thus, these studies showed that monocular depth cues, such as lighting, shadows, shading and texture, can influence depth perception.

About lighting, Lindemann et al. [16] focused on the influence of illumination models, such as Lambertian model, and found that the shading model influences the perceived depth. Others studied the effect of lighting depending on the kind of light, such as Langer and Bulthoff did [14] [15]. They showed that not only lighting direction but also shading can improve depth perception. The shading property of a virtual object is define by its material and its reflectance, thus shading might be considered as a depth cue resulting from lighting and texture, and similar to the shadowing depth cue. Diaz et al. demonstrated the importance of specular highlights for communicating depth information of a virtual object [7]. Moreover, shadow rendering on virtual objects can also increase the objects' presence [23]. Finally, another way to understand shading is to distinguish two different "shadow depth cues", the first being shadowing, meaning the cast shadows by the object on the others, and the second being shading, meaning the shadows present on the surface of the object [18]. Regarding texture, Mehrabi et al. [18] found that objects with smooth textures (less details) are interpreted as being farther away than objects with detailed texture, thus this cue can also influence depth perception. Moreover, Berbaum et al. found that interactions between shadow and materials in simulated lighting affect depth perceptions [3]. To conclude, these studies confirm that lighting, shading and texture can improve depth perception.

Regarding the eye-tracking technology, Feit et al. [10] studied its limitations to understand its functionality extend and usage. They found that the accuracy and precision varied not only between users (especially if they wore glasses or contact lenses), but also because of different lighting conditions. First, they observed that eye-tracking tends to have large variability in accuracy (which, in our study, justifies the use of depth cues to improve eye tracking precision); and, they found that in environments with greater luminance, eye tracking measures were more accurate. Finally, regarding the eye-tracking device precision of measurement, Antonya [2] studied the relation between the accuracy of the measure and the distance between the observer and the stared object - but all the studied distances were into the personal space. The targeted objects were projected images, thus the eye-tracking device used was similar to an augmented reality head-mounted display (as the user is able to see both the real world and virtual objects). The results indicated that the farther the objects were, the higher the relative error for the depth measure was.

\section{Methodology}

Our methodology consisted in comparing the distance measured by the eye-tracking device in the depth axis under varying conditions which, according to the literature, affect the depth perception, and thus potentially the depth measure. We used here the MagicLeap, an augmented reality device with an integrated eye-tracking system based on front-facing cameras, but the experiments could have been done with another device with such similar system. The MagicLeap captures the movements of each eye, and then, from the information gathered, calculates the gaze fixation point at run-time, represented as a three-dimensional vector $(\mathrm{X}, \mathrm{Y}, \mathrm{Z})$. The fixation point data represents the real-time gaze positions, which can be re-contextualised in the 3D world space (global coordinates), for obtaining the relevant information regarding the depth measure of the user's gaze.

\subsection{Preliminary study on eye-tracking measure}

Before focusing on depth, we conducted a preliminary experiment to confirm that measure errors occur more likely in this axis (Z) rather than in the horizontal (X) and vertical (Y) ones. To evaluate this assumption, we built a scenario where the users had to observe three virtual cubes that were appearing successively, all in a different position: one "top-left" cube, C1 (negative X value), one "centered" cube, C2 (X value: 0), and one "bottom-right" cube, C3 (positive $X$ value), all at a different depth, ordered from the closest to the farthest from the user's perspective. The user's eyes were located at the origin $(0,0,0)$ and the cubes at $(-1.0,1.0,0.5)$ for $\mathrm{C} 1,(0,0,1.0)$ for $\mathrm{C} 2$ and $(1.0,-1.0,1.5)$ for $\mathrm{C} 3$. In Fig. 1, the cubes are represented by purple crosses $(\mathrm{C})$ and the user by a green cross (U). This figure shows the measures obtained in the X-Z plane, as the results in the Y-axis did not present significant errors. Thus, we can observe that, while in the $\mathrm{X}$-axis the majority of the measures are correctly located in a range between $-1.5 \mathrm{~m}$ and $1.5 \mathrm{~m}$, in the $\mathrm{Z}$-axis (depth axis) they went up to $7 \mathrm{~m}$ instead of being between 0 and $2 \mathrm{~m}$ maximum. In this way, our initial assumption is confirmed, justifying the paper aim.

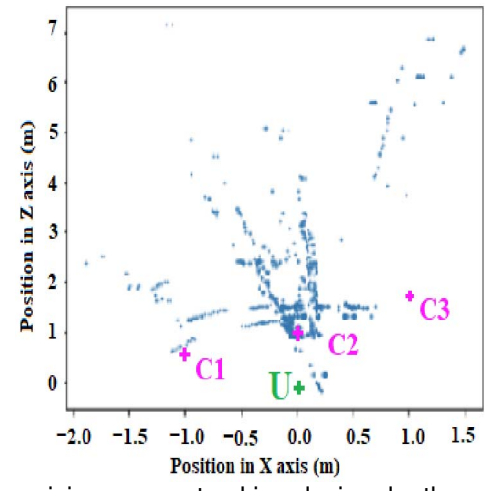

Figure 1: Imprecision on eye-tracking device depth measures ( $Z$ axis)

\subsection{Considered depth cues and hypotheses}

To study the influence of depth cues on the precision of eye-tracking measurements, lighting/shadowing and texture gradient were chosen into the list given by Mehrabi et al. [18]. This, because they are regarded as two of the most important monocular depth cues [18], and because they are reproducible on both virtual and real objects, which is ideal in an augmented reality environment. Then, our first hypothesis was that the light condition that had a major visual effect on shading would increase the precision of the depth measurement. Our second one stated that the texture condition with a major visual effect on shading would also increase the precision of the depth measurement. According to these hypotheses, we built an experiment for evaluating the lighting and texture effects based on the variation of such conditions. The lighting set-ups we chose were diffuse (omnidirectional) light and spot lights, whereas for the texture set-ups it was a high-smoothness texture and a polystyrene-like texture. Thus, spot lights and polystyrene-like texture were expected to improve depth measurement since they give better shading conditions.

\section{EXPERIMENT}

In this study, 20 subjects participated with an average age of 21.1 \pm 3.6 ( 14 male and 6 female). People with vision problems did not participate since these may influence depth perception [10], and depth measure. This was confirmed since they were not able to correctly fulfill the mandatory device calibration. Finally, two users raised some issues for performing the task during the experiment, thus we had to discard their results, which, indeed, were incoherent.

Each user, sitting on a chair, had to stare at an object, placed into his foveal vision, during 30 seconds. This was always at the same position: from the observer's perspective, at $1.5 \mathrm{~m}$ of distance in $\mathrm{Z}$, at $0 \mathrm{~m}$ in $\mathrm{X}$ and at the same $\mathrm{Y}$ of the gaze, about $1 \mathrm{~m}$ (i.e. right in front of $\mathrm{him} / \mathrm{her})$. In the world coordinates, the observer was located at (1.25, $1,-1.5)$ and the object at $(1.25,1,0)$. Fig. 2 gives schematic views of the experiment and Fig. 4 shows our implementation. Then, to study the effect of lighting and of texture, independently and combined, we built six scenarios. In each of them, the task was the one described above, and the object had always the same size, volume and shape (a sphere of $20 \mathrm{~cm}$ diameter). Then, in four scenarios, the sphere 
was virtual and designed depending on the varying conditions of light and texture (see Fig. 3). In addition, two scenarios with a real object (texture fixed, polystyrene) and real lights (varying, diffuse and spot) were considered, to obtain comparative measures (see Fig. 4). Hereafter the notion x-Nature-(Texture)-Light will be used to refer to a scenario, where $\mathrm{x}$ is its attributed number. The scenarios 1-Real-Diffuse and 4-Real-Spot share the same real object (and its texture) but under varying lighting conditions (diffuse VS spot). The four other scenarios used objects coming from the augmented reality device. Scenarios 2-AR-Smooth-Diffuse and 3-AR-Poly-Diffuse shared the same condition of diffuse lights but differed on the texture (smooth VS polystyrene), whereas the scenarios 5-AR-Smooth-Spot and 6-AR-Poly-Spot shared the same condition of spot lights and differed in the texture as well. Likewise, these scenarios may also be grouped by pairs where the varying condition is the light under a shared condition of texture, e.g 2-AR-Smooth-Diffuse with 5-ARSmooth-Spot. Finally, the eye-tracking measures were taken for each of the six scenarios for each user, thus we did 120 measurement sessions, and each session gave approximately 2500 measures.

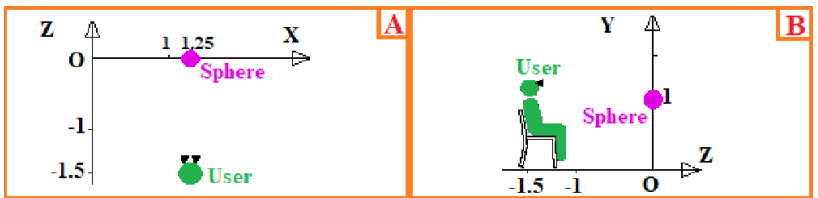

Figure 2: Experiment views, distances in $\mathrm{m:} \mathrm{A)} \mathrm{top} \mathrm{view} \mathrm{B}$ ) right view

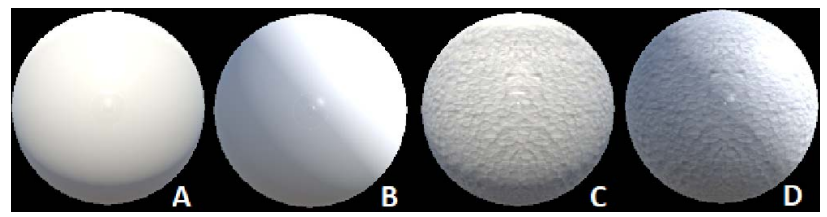

Figure 3: Virtual spheres in the experiments: A) 2-AR-Smooth-Diffuse - B) 5-AR-Smooth-Spot - C) 3-AR-Poly-Diffuse - D) 6-AR-Poly-Spot

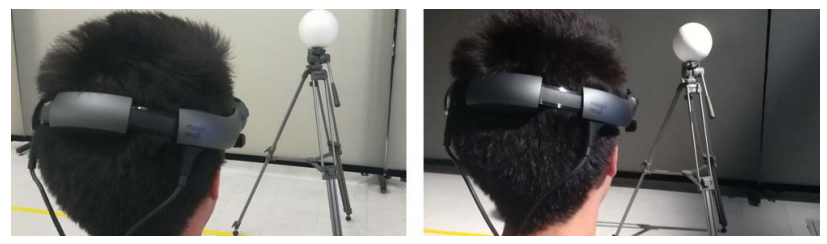

Figure 4: Scenarios with real object: diffuse light on left, spot on right

\section{Results}

Eye-tracking data collected in this experiment is presented in the next figures. It is important to note that, in the following graphs, the horizontal axis represents the measured values in $\mathrm{X}$ (horizontal position) and the vertical axis, in $\mathrm{Z}$ (depth position). Moreover, in world coordinates, the real and exact position of the targeted object was (x:1.5m, y:1.2 m, z:0m) for all the scenarios - position that is indicated by a purple cross in the next figures. Additionally, since more than 2500 positions were collected by participant and by scenario, we filtered the data by removing the measures located out of the ranges of the second standard deviation of the measures for each user in each scenario (except in Fig. 7). These statistical indicators allowed us to identify the outlying data.

Then, for each participant, we considered the measures obtained under the six scenarios to analyse the effect of each depth cue independently. Fig. 5 shows an example of these visualisations for one user, in this case, to understand the effect of lighting. Each graph of this figure shows 2 comparable scenarios: one with a diffuse light condition (blue) and another with a spot light condition (orange). The left graph shows the real sphere scenarios (1-RealDiffuse, 4-Real-Spot), the middle graph displays smooth-textured virtual spheres scenarios (2-AR-Smooth-Diffuse, 5-AR-SmoothSpot) and the right graph the polystyrene-like texture virtual spheres scenarios (3-AR-Poly-Diffuse, 6-AR-Poly-Spot). Similarly, Fig. 6 shows an example of the measures obtained for an user in the virtual object scenarios (2-AR-Smooth-Diffuse, 3-AR-Poly-Diffuse, 5-ARSmooth-Spot and 6-AR-Poly-Spot) under texture gradient varying conditions. The graph in the left shows the diffuse light scenarios (2-AR-Smooth-Diffuse, 3-AR-Poly-Diffuse) and the one in the right shows the spot light scenarios (5-AR-Smooth-Spot, 6-AR-PolySpot). The two scenarios with the real sphere cannot be graphed since in both of them the same real sphere (with the same texture) was used. Finally, Fig. 7 and Fig. 8 summarise all the data - for all conditions and users (with and without outlying data, respectively).

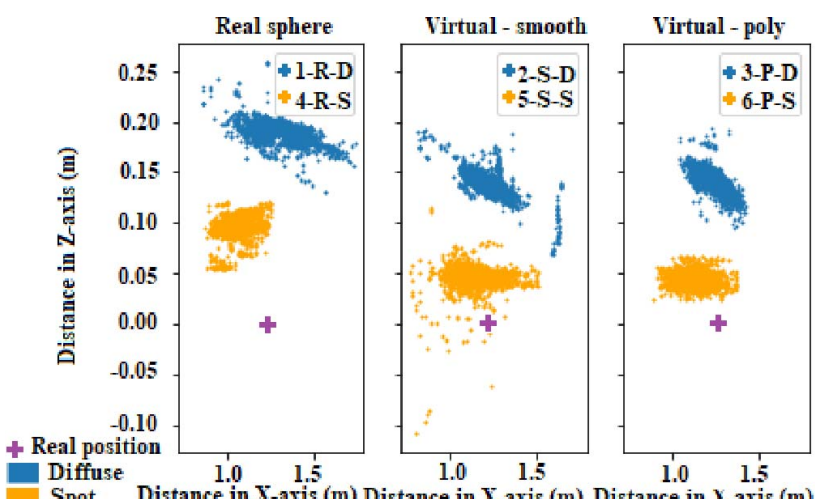

Figure 5: Depth measures, for one user, grouped by similar conditions of texture and under light varying condition

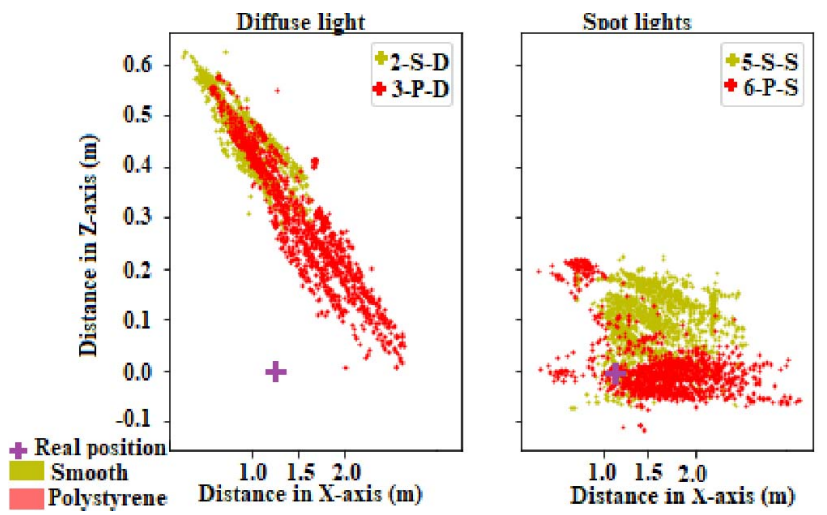

Figure 6: Depth measures, for one user, grouped by similar conditions of light and under texture varying condition

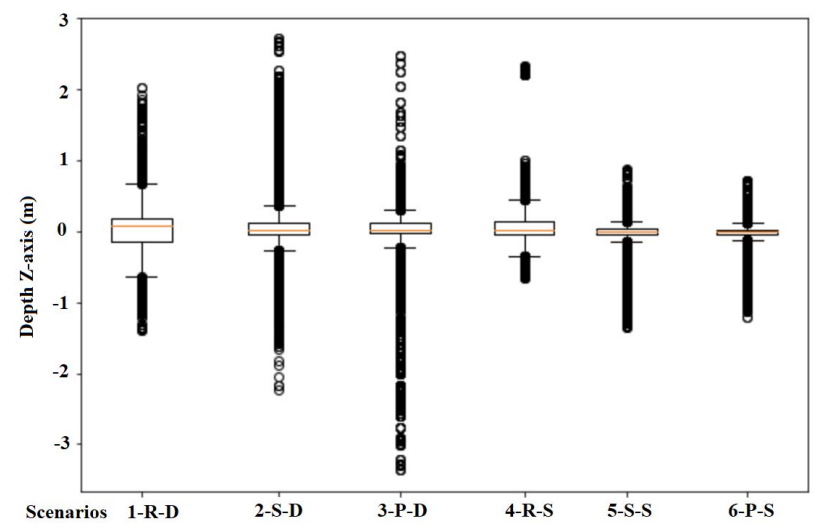

Figure 7: Box plot with outlying data for the 18 users under the 6 conditions: 1-Real-Diffuse, 2-AR-Smooth-Diffuse, 3-AR-Poly-Diffuse, 4-Real-Spot, 5-AR-Smooth-Spot, 6-AR-Poly-Spot 


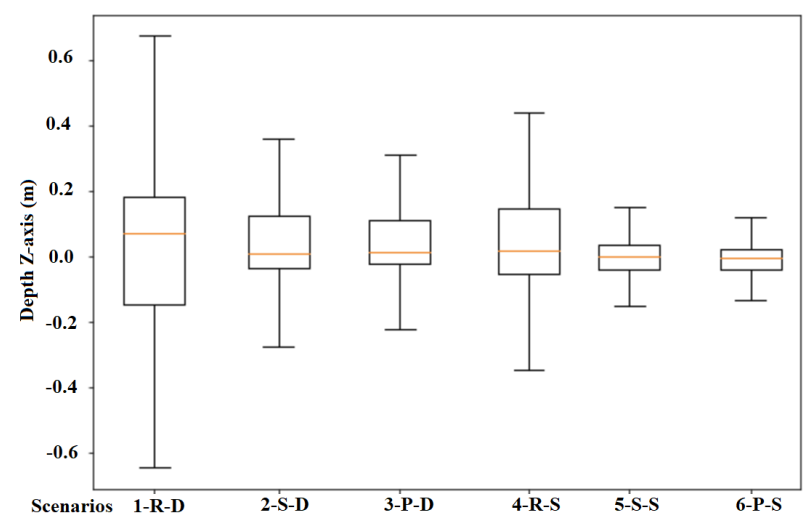

Figure 8: Box plot (no outlying data) for the 18 users under the 6 conditions: 1-Real-Diffuse, 2-AR-Smooth-Diffuse, 3-AR-Poly-Diffuse, 4-Real-Spot, 5-AR-Smooth-Spot, 6-AR-Poly-Spot

\section{Discussion}

First, we can observe graphically the results, when considering only one user. In Fig. 5, regardless of the texture conditions (real sphere, smooth texture, polystyrene-like texture), depth measures in the spot light scenarios (4-Real-Spot, 5-AR-Smooth-Spot, 6-AR-PolySpot) are more precise and accurate than in the ones in the diffuse light conditions. Moreover, the expected result being $0.0 \mathrm{~m}$ (the object is at z:0, $1.5 \mathrm{~m}$ away from the user, see Fig. 2), the data from the scenarios with diffuse light conditions (1-Real-Diffuse, 2-ARSmooth-Diffuse, 3-AR-Poly-Diffuse) show farther depth measures, about $10 \mathrm{~cm}$ of difference regarding spot light scenarios (z:0.20 for blue points vs 0.10 for orange ones). Such results are coherent with the literature, since it is mentioned that observers tend to overestimate distance in the personal space [9]. About the texture, it seems that the polystyrene one has a positive effect when combined to the spot lights, since scenario 6 has the more precise measures here. To conclude, these results tend to confirm that lighting, as a depth cue, can improve depth measure precision, when a lighting that generates more shading effect on the objects, such as spot light, are used.

Regarding Fig. 6, we can observe, in its right part, that rougher textures, which generate more shading effect on the objects, tend to allow obtaining more precise results, since measures from scenario 6-AR-Poly-Spot are closer to $0.0 \mathrm{~m}$ than the ones from scenario 5AR-Smooth-Spot. However, the results on the left do not indicate the same effect, between the scenarios 2-AR-Smooth-Diffuse and 3-ARPoly-Diffuse. Thus, the effect of the texture as a depth cue seems to be lower than the effect previously observed of lighting. It seems that it may have an effect only when combined to another depth cue, here lighting, on spot light condition. Indeed, in accordance with Fig. 5, we can observe that the scenario where the best results have been collected for this user (i.e. data with less dispersion and centered in the expected value z:0.0) is scenario 6-AR-Poly-Spot.

Still graphically, we can interpret the results considering all the users. The results shown in Fig. 7 indicate that, independently of the texture, spot light scenarios (4-Real-Spot, 5-AR-Smooth-Spot, 6-AR-Poly-Spot) gave less outlying data (black circles here) than scenarios with diffuse lights (1-Real-Diffuse, 2-AR-Smooth-Diffuse, 3-AR-Poly-Diffuse). This is relevant, as it confirms, for various users, how lighting increases the accuracy of the eye-tracking depth measure, by reducing the number of outliers. In Fig. 8 - data without the outliers, we can observe that the highest IQR (interquartile range) and st. dev. (standard deviation) are for scenarios with real objects, 1-Real-Diffuse and 4-Real-Spot. This insight was not expected, but might be explained by the fact that the participants may have focused more when looking at a virtual object than a real one, like in quotidian life when people pay more attention to cellphones or screens rather than other "real" objects. Additionally, by comparing results from scenarios 2-AR-Smooth-Diffuse and 3-AR-Poly-Diffuse with scenarios 5-AR-Smooth-Spot and 6-AR-Poly-Spot, we can observe that the texture allowed reducing the IQR size. Similarly, the effect of lighting to reduce the IQR size can also be noted by comparing the results from scenarios 1-Real-Diffuse and 4-Real-Spot, 2-ARSmooth-Diffuse and 5-AR-Smooth-Spot, and 3-AR-Poly-Diffuse and 6-AR-Poly-Spot. Finally, as expected and also in consonance with the previous results for one user, the measurement accuracy and precision (and the standard deviation) had the greatest improvement when combining both lighting and texture shading-favorable conditions (6-AR-Poly-Spot).

Then, these results can be analysed statistically. First, note that, for performing this analysis, we used the mean value obtained by each user in each scenario, instead of using all the measures of each session (about 2500 measures for one user under one light and texture condition). We could use the means here since all the comparisons, one for each condition, between the variance of the measures of each subject, were not significantly different. Additionally, note that this data followed a normal distribution for each scenario, since all the linear equations in the normal probability plots we obtained had a correlation coefficient squared higher than 0.98. Additionally, we applied the Levene's test on our data, which showed the homogeneity of the variance between the scenarios, on a level of significance of $\alpha=0.01$. Finally, since the observed factors were the light, the texture and the nature of the object, all were independent. Thus, the conditions for using a two-way ANOVA could have been ensured. However, as all the subjects participated to all the scenarios, our experiment was a case of repeated measures, thus we conducted a two-way repeated measures ANOVA.

According to the validation of these required assumptions, we ran two two-way repeated measures ANOVA. One ANOVA on the data of the scenarios with virtual objects $(2,3,5$ and 6$)$, to study the effect of the two factors light and texture, and then another ANOVA on the data of the scenarios with real object (1-Real-Diffuse, 4Real-Spot) and their associated scenarios (3-AR-Poly-Diffuse, 6AR-Poly-Spot), to study the effect of the object nature. The results of the latter revealed that the nature factor has not a significant effect on the depth measure ( $\mathrm{p}$-value $=0.85$ ). About the effects of lighting and texture, the results from the first ANOVA confirmed that lighting does have a significant effect on the measure of depth, with a p-value of 0.014 on a level of significance of $\alpha=0.05$. On the other hand, and, as expected thanks our first graphical analyses, it seems that the texture factor has not a significant effect on the depth measure, with a p-value of 0.65 . Moreover, the combined effect of light and texture seems to be not significant, with a p-value of 0.39 .

To resume, these analyses showed that light and texture depth cues could have a positive effect on depth measure. First, it appears that they can reduce the dispersion of the measures, which can help for standardising the measures taken among observers, making more uniform depth measurements. Then, the graphical analyses suggested that lighting has an effect on the precision of depth measure, and that this effect was more important than the one of the texture, which seemed to be limited to the scenario with the spot light condition. Finally, statistical analyses confirmed these results: the lighting does have statistically a significant effect on the depth measures, whereas the texture and the nature of the object do not have a significant effect.

\section{CONCLUSIONS AND FUTURE WORK}

To conclude, our initial hypotheses were confirmed thanks to this study: depth cues (lighting and texture here) which improve depth perception for the observers, can also improve the precision of the depth measurement, in eye-tracking devices with an eye-tracking mechanism similar to the MagicLeap. A first analysis of the results showed that both lighting and texture had a positive effect on improving this measurement. Nonetheless, statistically, the influence of lighting resulted in being significant whereas the one of texture was 
not. Thus, the latter might be only an "auxiliary" depth cue, meaning that it could be partially useful, only when combined to other depth cues. Moreover, thanks to this study, it has been possible to notice that these two depth cues also help to improve depth measurement precision by reducing the standard deviation between the measures taken from different users, thus giving more consistency for eyetracking depth computation. This may reduce the risk of highly imprecise measurements and may improve the user experience when this technology is used for this purpose. As future work, it could be also interesting to consider the effect of the real lightening on the environment brightness since it changes the pupil size, which in turn can change accommodation and distance perception [11]. Moreover, we could study how inconsistencies between the shading on virtual objects and the real lighting might also trouble the depth perception. It would also be interesting to make another study with still the same depth cues chosen here but with varying distance, in particular out of the personal space (more than $2 \mathrm{~m}$ ), to expand our scope. Finally, it could be interesting to repeat the experiment with other depth cues, and to analyse if they can also improve depth measure precision and accuracy.

\section{REFERENCES}

[1] R. S. Allison, B. J. Gillam, and E. Vecellio. Binocular depth discrimination and estimation beyond interaction space. Journal of Vision, 9(1):10-10, 01 2009. doi: 10.1167/9.1.10

[2] C. Antonya. Accuracy of gaze point estimation in immersive $3 \mathrm{~d}$ interaction interface based on eye tracking. In 2012 12th International Conference on Control Automation Robotics Vision (ICARCV), pp. 1125-1129, Dec 2012. doi: 10.1109/ICARCV.2012.6485315

[3] K. Berbaum, T. Bever, and C. S. Chung. Extending the perception of shape from known to unknown shading. Perception, 13(4):479-488, 1984. PMID: 6527935. doi: 10.1068/p130479

[4] D. A. Bowman, D. Koller, and L. F. Hodges. Travel in immersive virtual environments: an evaluation of viewpoint motion control techniques. In Proceedings of IEEE 1997 Annual International Symposium on Virtual Reality, pp. 45-52, March 1997. doi: 10.1109/VRAIS.1997.583043

[5] H. Choi, B. Cho, K. Masamune, M. Hashizume, and J. Hong. An effective visualization technique for depth perception in augmented reality-based surgical navigation. The international journal of medical robotics + computer assisted surgery : MRCAS, 12, 05 2015. doi: 10 . $1002 /$ rcs. 1657

[6] J. Cutting and P. Vishton. Perceiving layout and knowing distances: The interaction, relative potency, and contextual use of different information about depth, vol. 5, pp. 69-177. 011995.

[7] C. Diaz, M. Walker, D. A. Szafir, and D. Szafir. Designing for depth perceptions in augmented reality. In 2017 IEEE International Symposium on Mixed and Augmented Reality (ISMAR), pp. 111-122, Oct 2017. doi: 10.1109/ISMAR.2017.28

[8] J. Dorado, J.-R. Chardonnet, P. Figueroa, F. Merienne, and J. Hernandez. A perceptual calibration method to ameliorate the phenomenon of non-size-constancy in hetereogeneous vr displays. 06 2018. doi: 10. 29007/sxjn

[9] F. El Jamiy and R. Marsh. A survey on depth perception in head mounted displays: Distance estimation in virtual reality, augmented reality and mixed reality. IET Image Processing, 13, 01 2019. doi: 10. 1049/iet-ipr.2018.5920

[10] A. M. Feit, S. Williams, A. Toledo, A. Paradiso, H. Kulkarni, S. Kane, and M. R. Morris. Toward everyday gaze input: Accuracy and precision of eye tracking and implications for design. In Proceedings of the 2017 CHI Conference on Human Factors in Computing Systems, CHI '17, pp. 1118-1130. ACM, New York, NY, USA, 2017. doi: 10.1145/3025453. 3025599

[11] R. Hennessy, T. Iida, K. Shiina, and H. Leibowitz. The effect of pupil size on accommodation. Vision Research, 16:587-589, 1976. doi: 10. 1016/0042-6989(76)90004-3

[12] W. Jaschinski, S. Jainta, J. Hoormann, and N. Walper. Objective vs subjective measurements of dark vergence. Ophthalmic \& physiological optics : the journal of the British College of Ophthalmic Opticians (Optometrists), 27:85-92, 02 2007. doi: 10.1111/j.1475-1313.2006. 00448.x

[13] E. Kruijff, J. E. Swan, and S. Feiner. Perceptual issues in augmented reality revisited. In 2010 IEEE International Symposium on Mixed and Augmented Reality, pp. 3-12, Oct 2010. doi: 10.1109/ISMAR.2010. 5643530

[14] M. Langer and H. Bülthoff. A prior for global convexity in local shapefrom-shading. Perception, 30:403-410, 02 2001. doi: 10.1068/p3178

[15] M. S. Langer and H. H. Bülthoff. Depth discrimination from shading under diffuse lighting. Perception, 29(6):649-660, 2000. PMID: 11040949. doi: $10.1068 / \mathrm{p} 3060$

[16] F. Lindemann and T. Ropinski. About the influence of illumination models on image comprehension in direct volume rendering. IEEE Transactions on Visualization and Computer Graphics, 17(12):19221931, Dec 2011. doi: 10.1109/TVCG.2011.161

[17] M. Livingston, A. Dey, C. Sandor, and B. Thomas. Pursuit of "X-Ray Vision" for Augmented Reality. 09 2012. doi: 10.1007/978-1-4614 -4205-9_4

[18] M. Mehrabi, E. Peek, B. Wünsche, and C. Lutteroth. Making 3d work: a classification of visual depth cues, $3 \mathrm{~d}$ display technologies and their applications. pp. 91-100, 012013.

[19] M. Mikkola, A. Boev, and A. Gotchev. Relative importance of depth cues on portable autostereoscopic display. MoViD'10 - Proceedings of the 2010 ACM Workshop on Mobile Video Delivery, Co-located with ACM Multimedia 2010, 01 2010. doi: 10.1145/1878022.1878038

[20] S. Reichelt, R. Haeussler, G. Fütterer, and N. Leister. Depth cues in human visual perception and their realization in $3 \mathrm{~d}$ displays. vol. 7690 , 04 2010. doi: 10.1117/12.850094

[21] S. Schmidt, G. Bruder, and F. Steinicke. Illusion of depth in spatial augmented reality. pp. 1-6, 03 2016. doi: 10.1109/PERCAR.2016. 7562417

[22] S. Schmidt, G. Bruder, and F. Steinicke. Moving towards consistent depth perception in stereoscopic projection-based augmented reality. In Proceedings of the 27th International Conference on Artificial Reality and Telexistence and 22Nd Eurographics Symposium on Virtual Environments, ICAT-EGVE '17, pp. 161-168. Eurographics Association, Goslar Germany, Germany, 2017.

[23] N. Sugano, H. Kato, and K. Tachibana. The effects of shadow representation of virtual objects in augmented reality. In The Second IEEE and ACM International Symposium on Mixed and Augmented Reality, 2003. Proceedings., pp. 76-83, Oct 2003. doi: 10.1109/ISMAR.2003. 1240690

[24] J. E. Swan, A. Jones, E. Kolstad, M. A. Livingston, and H. S. Smallman. Egocentric depth judgments in optical, see-through augmented reality. IEEE Transactions on Visualization and Computer Graphics, 13(3):429-442, May 2007. doi: 10.1109/TVCG.2007.1035

[25] L. R. Wanger, J. A. Ferwerda, and D. P. Greenberg. Perceiving spatial relationships in computer-generated images. IEEE Computer Graphics and Applications, 12(3):44-58, May 1992. doi: 10.1109/38.135913 